\title{
Creencias y expectativas respecto de la consulta médica: Un análisis de diferencias culturales
}

\author{
FELIPE G. CASTRO \\ University of California, Los Angeles \\ JESÚS RODRÍGUEZ \\ Universidad de Alicante

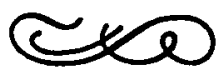 \\ Resumen
}

Se compararon 102 mujeres del área metropolitana de los Angeles divididas en tres grupos según su grado de aculturación (mejico-americanas vs. anglo-americanas). Las variables consideradas se refirieron a las expectativas respecto al médico en contextos terapéuticos. Se consideraron las puntuaciones de tres medidas: La puntuación total en expectativas respecto del médico, las puntuaciones respecto a las expectativas referentes al comportamiento afectivo del médico y las puntuaciones respecto a las expectativas de tratamiento concreto. Los análisis de los resultados y el control de diversas variables tales como edad, gravedad de la enfermedad, etc., muestran la ausencia de diferencias interculturales en lo que las mujeres de la muestra esperan del médico. Si estos resultados se confirman obligarian a identificar más claramente cuándo son importantes las diferencias de aculturación y cuándo no lo son.

\section{Abstract}

102 women coming from los Angeles were divided according their aculturation characteristics (mexico-american vs. anglo-american) and compared in terms of their expectations with respect to the physicians' practices. Several variables such as age, illness, etc., were controlled. The results show a lack of intercultural differences in the expectations of women. These results could imply a reconsideration of the role of cultural differences in the physician-patient relationships.

Agradecimientos: El trabajo del segundo autor fue facilitado por una beca de investigación otorgada por la Dirección General de Política Científica de la Consellería de Cultura, Educación y Ciencia, de la Generalitat Valenciana, que posibilitó su estancia en la Universidad de California, en Los Angeles. El segundo autor expresa, igualmente su reconocimiento al Profesor Bertram H. Raven, Director del Departamento del Psicología de UCLA, al Profesor Amado Padilla, Director del Spanish Speaking Mental Health Research Center, su ayuda y apoyo durante su estancia en tal Departamento y Centro.

Dirección del autor: J. Rodríguez Marín, Fac. de Medicina, Univ. de Alicante, Alicante. 
A menudo los médicos han notado que los pacientes, particularmente los de clase baja o los pacientes latinos de bajo nivel de aculturación en países como EE. UU., dejan de cumplir con frecuencia el tratamiento prescrito por el médico y/o no están motivados para dedicarse al cuidado de su propia salud. Una posible explicación de este problema sobre la base de rasgos de personalidad presenta al paciente como apático y falto de deseo para cambiar (Davis, 1966). Esta concepción "victimiza» al paciente, haciendo hincapié en su papel en el proceso de observancia o cumplimiento del tratamiento, mientras que, por el contrario, quita importancia al papel del médico en el problema del incumplimiento. Se trata, por tanto, de una explicación coherente con el marco conceptual del llamado modelo «biomédico" en cuyo seno se acopla perfectamente el modelo parsoniano del juego de papeles sociales en la interacción médico-paciente (Parsons, 1951; 1958; 1975; Mechanic, 1968).

Sin embargo, la aparición y consolidación reciente de otro modelo más amplio y comprensivo sobre la salud, el modelo biopsicosocial, puede aportar una concepción más amplia y precisa de este problema del incumplimiento o falta de observancia del tratamiento y de la ausencia o presencia de motivación en el cuidado de la propia salud. Tal concepción requiere un análisis de las expectativas y conductas en la diada médico-paciente en cuanto éstas ocurren dentro del contexto del sistema actual de cuidado médico (Dimatteo y Dinicola, 1982; Dimatteo y Friedman, 1982). Las expectativas de mayor frecuencia sobre los roles del sistema médico tradicional (modelo biomédico) sesgan el papel del médico hacia la provisión de un tratamiento que implique el alivio o supresión de los síntomas, y en su caso, de las causas de una enfermedad en el plazo más breve posible, más que hacia una actuación dirigida a una promoción de la salud: un proceso de valoración de la salud, de educación para la salud y de motivación para el autocuidado de la salud.

De forma similar, desde la perspectiva del paciente, las expectativas de recibir pasivamente una cura por parte del médico y de obtener un alivio o supresión inmediatos de los síntomas, van en contra de un control activo de su salud que permita, a más largo plazo, la prevención de desórdenes futuros y el aumento de su salud. La motivación para una implicación en el autocuidado activo se ve desalentada en situaciones en las que el médico asume un papel activo en la prescripción de medicamentos para eliminar los síntomas y en las que el paciente espera pasivamente ser tratado o curado por el médico. En estas situaciones, los pacientes sólo están motivados para tomar los medicamentos prescritos y usualmente lo hacen así sólo hasta que obtienen el alivio (en muchos casos aparente) de los síntomas.

La motivación para participar en el cuidado de la propia salud es un estado cognitivo con antecedentes múltiples. Las personas se ven muy motivadas para realizar unas tareas como resultado de factores interpersonales, tales como la influencia social de otra persona o personas que consideran importantes para ellas (Dimatteo y Dinicola, 1982; Ajzen y Fishbein, 1980) y como resultado de factores cognitivos, creeencias, expectativas positivas de que un resultado deseado resulte de realizar una tarea particular (Ajzen y Fishbein, 1980). En el marco médico, el médico, como una figura de autoridad, puede aumentar esa motivación a través de la influencia interpersonal y proporcionando expectativas positivas, reforzando las creencias de 
que se obtendrán buenos resultados ejecutando determinadas conductas (Jaspars, King y Pendleton, 1983; Rodin y Janis, 1979). El problema de cómo aumentar la motivación implica saber más acerca de las expectativas de los pacientes, de tal modo que se puedan alentar las expectativas congruentes con una perspectiva de promoción de la salud, y de tal modo que se puedan modificar las expectativas que entren en conflicto con tal perspectiva.

Los pacientes pueden presentar diferencias, adquiridas socioculturalmente, respecto a las expectativas que tienen sobre la actuación del médico, basadas en sus creencias sobre la salud y la enfermedad y basadas también en su concepción de cuáles son las conductas de rol apropiadas en el encuentro medio-paciente (Weis y Davis, 1983; Dimatteo Dinicola, 1982; Lurie y Lawrence, 1971; Harwood, 1981), que son básicamente construidas como una normativa social emanada del grupo de personas o grupos importantes para el sujeto y que constituyen, en gran parte, las posiciones de referencia en los segmentos del rol (Ajzen y Fishbein, 1980; Bochner, 1983). $\mathrm{El}$ paciente, como un consumidor de servicios de salud, tiene algunas expectativas de cómo actuará el médico. Necesitamos más información sobre tales expectativas referidas a diversos tipos de pacientes y sobre si estas expectativas son congruentes o no con la perspectiva de promoción de la salud.

Este estudio examina las expectativas que, en el cuidado de su salud, tienen diversas mujeres respecto al médico, y en qué medida estas expectativas pueden ser compatibles o incompatibles con una perspectiva de promoción de la salud. El estudio considera si tales expectativas difieren interculturalmente entre mujeres anglo-americanas, méjico-americanas, y si el locus de responsabilidad de salud (interno o externo) está relacionado con esas expectativas.

Del Vechio Good, y Nasi (1983) han subrayado la necesidad de investigaciones más sistemáticas sobre lo que piden y esperan de sus médicos los pacientes de clínicas ambulatorias y la necesidad de desarrollar, asimismo, una escala de expectativas de los pacientes respecto al cuidado primario. Un análisis factorial de su escala proporcionó cinco categorías de expectativas del paciente: 1) tratamiento de problemas psicosociales, 2) explicaciones médicas, 3 ) comunicaciones de apoyo, 4) resultados de pruebas, y 5) ventilación emocional/legitimación. Otros investigadores han categorizado las expectativas de los pacientes en la clínica ambulatoria como: 1) «somáticas», es decir, el paciente desea un tratamiento concreto, tal como medicación o un análisis de sangre, una radiografía, etc., y 2) «cognitivas», es decir, el paciente desea información o una explicación médica (Koopman et al., 1984). Otros autores han subrayado la importancia que los pacientes en consulta ambulatoria ponen: 1) en la información (Acosta, 1980; Marin y Triandis, 1984), 2) en recibir cuidados de un médico que trabaje pausadamente y que alivie la ansiedad del paciente (Acosta, 1980; Mollerman et al., 1984), y 3) en que el médico se preocupe de reducir el estigma o embarazo asociado con padecer un problema de salud dado o someterse a un procedimiento médico concreto (Trotter, 1982).

Los mejicanos y otras personas latino-hispanas han sido descritos como una minoría étnica en los EE. UU.; muchos de ellos tienen expectativas culturales que pueden entrar en conflicto con las de los médicos que pertene- 
cen a la mayoría cultural angloamericana (Harwood, 1981). Se ha postulado que la medida en que la expectativa entra en conflicto entre el médico angloamericano y el paciente mejicano covaria inversamente con el nivel de aculturación de la persona de origen mejicano inmerso en el sistema sociocultural norteamericano (Cuellar et al., 1983). La persona de origen mejicano con baja aculturación ha sido caracterizada por tener una concepción del mundo «al viejo estilo», tradicional, o una cultura subjetiva (normas, valores, creencias, costumbres y conductas) tradicional mejicana (Arce y Torres, 1982; Triandis et al., 1982).

Por el contrario, a los méjico-americanos más aculturados se les ha supuesto una concepción del mundo más de acuerdo con la «cultura mayoritaria" en EE. UU. Estos méjico-americanos más aculturados tendrían un grado menor de identificación y participación en las actividades tradicionales mejicanas tales como: hablar español, observar las fiestas culturales y mantener valores, costumbres, creencias y conductas mejicanas (Harwood, 1981). El grado de «etnicidad», en cuanto indicado por el nivel de aculturación, es una variable moderadora importante indicativa de las diferencias intragrupos entre personas de origen mejicano (Padilla, 1980). También se ha subrayado la necesidad de más investigaciones en varios aspectos de estas diferencias intragrupos relacionadas con la aculturación (Padilla y Lindholm, 1984).

Las expectativas de cuidado médico que las personas de origen mejicano de baja aculturación tendrían respecto del médico pueden reflejar su mantenimiento de los valores o creencias culturales mejicanos tradicionales. Estos valores o creencias tradicionales han sido identificados como conteniendo: 1) una fuerte valoración de las relaciones interpersonales estrechas, 2) una valoración de la cooperación sobre la competición, 3) una valoración del logro en beneficio de la familia, 4) una orientación externa en locus de control, 5) un enfoque en los síntomas corporales en los casos de enfermedad, con poca atención a las causas psicosociales y 6) evitación de conflictos y limitación en la expresión de la ira o de conductas agresivas (Arce y Torres-Marullo, 1982).

Este patrón de expectativas, que puede describirse como un modelo tradicional mejicano, subraya los valores «alocéntricos», centrados en la familia. Tal orientación contrasta con el modelo cultural anglo-americano que, por comparación, subraya los valores individualistas, «idiocéntricos", tales como el individualismo, la competitividad, el locus interno de control, la disponibilidad para analizar los problemas desde un punto de vista psicológico y el consumismo. Tales valores son, culturalmente, más adaptativos para sobrevivir en las grandes corrientes del sistema social norteamericano (Marin y Triandis, 1984). Sin embargo, ambos modelos culturales parecen compartir las mismas pautas de conductas de rol correspondientes a la interacción de la diada médico-paciente, que corresponden al modelo más "clásico" descrito por Parsons (1958; 1975). Este modelo, aunque parece que ha empezado a cambiar al mismo tiempo que el modelo general de la salud/enfermedad pasando desde la perspectiva biomédico a la biopsicosocial todavía se puede observar en la mayoría de las interacciones en la consulta médica (Bochner, 1983).

Justamente como en el modelo parsoniano, el modelo cultural mejicano-tradicional pone al médico en el papel de proveedor de salud autorita- 
rio, pero supone que, además de proporcionar tratamientos médicos, "debería» mostrar preocupación por la salud y el bienestar de sus pacientes. En este modelo se supone que el médico debe interaccionar con el paciente de una forma autoritaria pero cálida, y mostrar una preocupación individualizada ("personalismo») (Trotter, 1982). Haciéndolo así, ayudaría también a aliviar la ansiedad del paciente, una pauta comportamental de apoyo con efectos curativos similares a los ofrecidos por los "curanderos", sanadores populares en el mundo hispano (Schreiber y Homiak, 1981). Sin embargo, este modelo no parece presentar muchas diferencias con en el que aparece en los trabajos citados por Jaspars, King y Pendleton (1983), en los cuales pacientes ingleses consideraban una consulta como «buena» aquella en la que se le proporcionaba un tratamiento efectivo, era aceptado como persona y sus quejas tomadas en serio, el médico prestaba atención a sus problemas, se tomaba tiempo suficiente para atenderle y mostraba comprensión.

El modelo cultural tradicional mejicano, con creencias somáticas acerca de la enfermedad, predice que el hecho de que la paciente de baja aculturación centre su atención básicamente en los síntomas corporales le conducirá a esperar que el médico le proporcione un tratamiento concreto y directivo. Tal tratamiento incluye hacer pruebas diagnósticas y recibir consejo médico acompañado de medicación para conseguir un alivio inmediato de los síntomas o la curación completa (Acosta, 1980; Arce y TorresMatrullo, 1982). De acuerdo con ello, los pacientes orientados tradicionalmente se sentirían insatisfechos si se les proporcionara "sólo" seguridad, apoyo psicológico o un programa de cambio de conducta, especialmente si tales cosas se les ofrecen en lugar de medicamentos. De forma semejante, el modelo cultural mejicano tradicional predice que los pacientes esperan recibir del médico un trato "personalizado" se sentirían insatisfechos si recibieran un trato impersonal, semejante a una relación de negocios, precipitada, brusca y fría (Schreiber y Homiak, 1981). Además, al incluir también una orientación valorativa de evitación del conflicto personal, tales pacientes, en el caso de quedar insatisfechos, dudarían en plantear cuestiones y en expresar su insatisfacción al médico, una figura de autoridad que generalmente se mantiene en alta estima y cuyo juicio es difícil desafiar (Acosta, 1981; Arce y Torres-Matrullo, 1982). Esa insatisfacción se produciría dentro del marco de la consulta médica, pero se manifestaría en el incumplimiento del paciente en cuanto a la toma de medicamentos o en el rechazo a volver a seguir un tratamiento ya prescrito.

El modelo cultural mejicano tradicional predice, asimismo, que una orientación de locus de control externo emparejado con una tendencia hacia la somatización, conduciría a tales pacientes a participar pasivamente en su propio tratamiento, mientras que el médico proporciona activamente el cuidado de la salud requerido. Esta expectativa activo-pasiva de los roles apropiados para el médico y para el paciente es antitética a una perspectiva de promoción de salud en la que el paciente es animado a participar como un colaborador activo y responsable junto con el médico en un programa de mantenimiento de la salud (Faber, 1982; Standard y Kaplun, 1983; Yamamoto et al., 1984; Marin y Triandis, 1984). Desde una perspectiva de promoción de la salud, la buena salud es consecuencia de la atención diaria que una persona presta a su bienestar, adoptando un estilo de vida saluda- 
ble. La atención a la salud es así, desde esta perspectiva, una actividad diaria más que un acontecimiento episódico centrado en un momento de crisis, que sólo merece atención en cuanto deteriora manifiestamente la salud (Standard y Kaplun, 1983; Leigh, 1983). Los pacientes mejicanos de orientación tradicional en tanto que guiados por tal "modelo médico clásico", de expectativas activas-para-médicos y pasivas-para-pacientes, quedarían insatisfechos con la acción de un médico que enfatizara la prevención primaria y la promoción de la salud. A tales pacientes no les gustaría que el médico les recomendara cambios de vida autodirigidos cuando eso fuera lo indicado. Igualmente, tales pacientes podrían no aceptar los incentivos motivacionales y la información sobre su salud para producir cambios comportamentales cuando el médico se los recomendara (Nyazema, 1984; Wells, Ware y Lewis, 1984).

En la medida en que los pacientes de origen mejicano de bajo nivel de aculturación actuarían bajo los supuestos de salud de ese modelo tradicional, del modelo médico clásico, no estarían preparados' para una actuación de promoción de la salud, sino que, por el contrario, esperarían que fuera el médico quien les proporcionara un tratamiento concreto, inmediato, centrado en los síntomas. Un tratamiento preventivo, que promocionara la salud subrayando el cambio de conducta, aparentemente entraría en conflicto con tales expectativas y podría engendrar insatisfacción y falta de observancia de las recomendaciones y prescripciones médicas.

En cualquier caso, las características del modelo cultural mejicano tradicional respecto a las expectativas de rol correspondientes a los papeles sociales de médico y paciente y, en general, a la parte del modelo que toca a las creencias sobre salud y enfermedad, no parecen diferir gran cosa de las de modelo clásico descrito por Parsons vigente en los EE. UU. de los años cincuenta, y vigente hoy en muchas partes diferentes del mundo occidental (Szasz y Hollender, 1956; Dimatteo y Friedman, 1982). Las diferencias más apreciables se refieren sobre todo a los componentes más emocionales de la relación interpersonal, tales como la simpatía, la relación cálida, «humana» y aparentemente menos «tecnológica» la preocupación por problemas personales y familiares distintos a los que corresponden a la enfermedad concreta, etc.Sin embargo, la dimensión actividad-pasividad (médico-paciente) y el énfasis en los aspectos somáticos que requieren tratamiento puntual, se mantienen como un factor común en ambos modelos culturales, tal como estos aparecen descritos por la bibliografía al respecto.

De acuerdo con la tesis de que las diferencias en los modelos culturales implican diferencias en las expectativas acerca de la actuación del médico en la consulta, se presentan dos hipótesis sobre las diferencias de grupo en la orientación del cuidado de salud en cuanto relacionado con una perspectiva de promoción de salud:

Hipótesis 1: Efectos de la etnicidad. Las mujeres de origen mejicano comparadas con las mujeres de origen anglo-americano exhibirán expectativas más fuertes (preferencias) de recibir cuidado afectivo, tratamiento concreto y corto y expectativas más débiles respecto al auto-cuidado de la salud.

Hipótesis 2: Responsabilidad sobre la propia salud. Las mujeres con bajo nivel de responsabilidad sobre su salud, comparadas con aquellas que tienen un alto nivel de responsabilidad sobre su salud, exhibirán expectativas más fuertes (preferencias) de recibir cuidado afectivo, y tratamiento con- 
creto y breve, y expectativas más débiles respecto al autocuidado de su salud.

\section{METODO}

\section{Sujetos}

Participaron en este estudio 102 mujeres del área metropolitana de Los Angeles. De ellas 30 asistían a una clínica médica de la comunidad, localizada en el este de Los Angeles, y que es utilizada fundamentalmente por pacientes latino/hispanos. Las otras 72 mujeres eran estudiantes de la Universidad de California en Los Angeles (UCLA). Treinta y dos de las mujeres universitarias eran de origen mejicano, y la mayoría de ellas se identificó como méjico-americanas, 40 eran de origen no-mejicano (ni hispano) y la mayoría de ellas se identificó como anglo-americanas.

\section{Procedimiento}

Cada sujeto fue entrevistada individualmente usando un guión de entrevista estructurada, el Inventario de Creencias y Conducta de Salud, específicamente construido para este estudio. Los formularios del protocolo de la entrevista en inglés y en español, conceptualmente equivalentes, fueron debidamente desarrollados y validados en un pequeño estudio piloto preliminar usando los métodos de desarrollo de instrumentos interculturales recomendados por Brislin (1970). Los sujetos pudieron elegir ser entrevistadas en inglés o en español.

Este estudio se centra sobre la sección de la entrevista estructurada que examina las expectativas que las pacientes tienen respecto del médico. La Escala de expectativas respecto del médico consiste en 14 ítems. Cada uno de ellos contiene la introducción: "Cuando voy a ver a un médico porque me encuentro enfermo, espero que el médico...", seguida por el ítem mismo, por ejemplo, «Pase algún tiempo hablando y se muestre amistoso». Se puede contestar a cada ítem mediante una escala de cinco puntos de nivel de expectativa: $0=$ nunca, $1=$ rara vez, $2=$ algunas veces, $3=$ a menudo y 4 = siempre.

Todos los sujetos cumplimentaron mediante la entrevista una hoja de información sociodemográfica, y además una versión de 19 ítems de la Escala de Aculturación para méjico-americanos (ARSMA) (Cuéllar y Harris, 1980). Cada ítem de la ARSMA puede contestarse también mediante una escala de cinco puntos, en la que 1 = orientado a la cultura mejicana, $3=$ bilingual/bicultural, y $5=$ orientado a la cultura norteamericana. Se utilizó la puntuación media basada en estos ítems para obtener una indicación del estatus de aculturación del sujeto. La puntuación de los sujetos méjico-americanos fue desde 1.05 hasta 4.16 , con una puntuación de 2.95 como mediana. La gama de puntuaciones de las mujeres anglo-americanas en la ARSMA fue desde 4.20 hasta 4.95 , así que no hubo solapamiento en la distribución de las puntuaciones obtenidas en esa escala por los dos grupos de sujetos. 
Durante el análisis de los datos, los sujetos fueron divididos en tres grupos: bajo, medio y alto, sobre la base de la puntuación obtenida por el sujeto en la ARSMA. Utilizando la mediana de las puntuaciones en esta escala, los sujetos de origen mejicano fueron categorizados en dos grupos: de aculturación baja y de aculturación media (bilingual/bicultural). Salvo nueve, el resto de las 32 mujeres universitarias de origen mejicano permanecieron en el grupo de «aculturación media», y salvo 10 el grupo de mujeres que asistían a la clínica ambulatoria permanecieron en el grupo de «baja aculturación».

Sobre la base de la reagrupación parcial mediante su puntuación en aculturación, se constituyeron los tres grupos para este estudio: 1) 31 mujeres de baja aculturación la mayoría de las cuales se identificó como «mejicanas»; 2) 31 mujeres de aculturación media, la mayoría de las cuales se identificó como "méjico-americanas», y 3) 40 mujeres, de aculturación obviamente alta, la mayoría de las cuales se identificó como «anglo-americanas».

Para los grupos de aculturación baja, media y alta las edades medias fueron $36.06,28.13$ y 19.28 respectivamente, $F(2,99)=21.08$, $p<.001$. Una prueba de Scheffe identificó al grupo de baja aculturación como significativamente más viejo que los otros dos grupos. Las puntuaciones medias en estatus de salud según autoinforme fueron, respectivamente, 4.44, $5.46 \mathrm{y}$ 6.69 respectivamente, cuando se valoró mediante una escala que iba desde (1) = muy enfermo, con incapacidad grave, hasta $(7)=$ muy sano, con gran energía, $F(2,90)=13,42, p<.05$. La prueba de Scheffe identificó a las mujeres de baja aculturación como significativamente más bajas en su estatus de salud que las de los otros dos grupos. Los tres grupos difirieron también entre sí en años de estancia en los EE. UU., con una media de 9.43, 13.39 y 14.13 años respectivamente, $F(2,89)=9.84, \mathrm{p}<.05$. La prueba de Scheffe identificó a las mujeres de baja aculturación como significativamente menos expuestas al sistema norteamericano de educación. Los tres grupos de mujeres no diferían en la cuantía de ingresos mensuales medios, en su procedencia rural-urbana, ni en el nivel de participación religiosa activa.

\section{ESCALAS}

Los 14 ítems para la «Escala de Expectativas respecto del médico» se produjeron a partir de unas entrevistas piloto de respuesta abierta con pacientes de origen mejicano en una clínica ambulatoria de la comunidad. Se obtuvieron ítems que cubrían expectativas para: 1) cuidado afectivo, «ayudarme a sentirme menos nerviosa por mi enfermedad"; 2) tratamiento concreto, «darme una receta para un medicamento»; 3) información médica, «darme información sobre mi enfermedad", y 4) preferencia por un tratamiento breve frente a uno comprensivo, "verme sólo una vez". Koopman, Eisenthal y Stoeckle (1984) se han referido al contenido de ítems en los dominios 2 y 3 , respectivamente, como formas «somática» y «cognitiva» de las expectativas dèl paciente.

El análisis de fiabilidad realizado sobre estos 14 ítems para todos los sujetos arrojó un coeficiente alfa de .70 . Este resultado indica que este con- 
junto de ítems tiene suficiente consistencia interna para ser usado como una escala. Esta escala es un índice de la intensidad de la expectativa de la paciente respecto de la consulta médica. Se llevó a cabo, además, un segundo análisis de fiabilidad para valorar las posibles propiedades de las 4 subescalas conceptuales. Tres ítems que registraban expectativas de cuidado afectivo produjeron un coeficiente alfa de .60 , y cuatro ítems que registraban expectativas de tratamiento concreto produjeron un coeficiente alfa de .69. Los ítems que registraban expectativas de información y preferencia por un tratamiento breve produjeron coeficientes de fiabilidad demasiado bajos para que pudiéramos pensar en usarlos como escalas. Los valores del coeficiente alfa para los primeros dos conjuntos de ítems proporcionaron apoyo para su uso como escalas que miden el deseo de un paciente de «cuidado afectivo" y de "tratamiento concreto" respectivamente.

La tabla I muestra el conjunto completo de ítems de la Escala de Expectativas respecto del médico. Los tres primeros ítems pertenecen a la subescala de "cuidado afectivo" y los ítems 4 y 7 constituyen la subescala de "tratamiento concreto".

TABLA I

Escala de expectativas respecto del médico e items de las subescalas

Cuando consulto al médico porque me siento enfermo, espero que el médico:

Subescala de Cuidado Afectivo (CA) (alfa $=.60)$

1. Pase un cierto tiempo hablando conmigo amistosamente.

2. Me ayude a sentirme menos nerviosa por mi enfermedad.

3. Me cure.

Subescala de Tratamento Concreto (TC) (alfa $=.69$ )

4. Ordene que me pongan unas inyecciones.

5. Me de una receta para unas medicinas.

6. Ordene un análisis de sangre.

7. Ordene un análisis de orina.

Items referidos a información

8. Me proporcione información sobre mi enfermedad.

9. Me enseñe nuevas maneras de tratar con mi problema.

10. Me ayude respecto a otros problemas, además de mi enfermedad.

Items referidos a tratamiento breve o comprehensivo

11. Me proporcione un alivio rápido de mis síntomas.

12. Me haga un reconomiento físico completo.

13. Me vea sólo una vez, sin necesidad de nuevas visitas.

14. Me pida que vuelva para una visita de seguimiento.

\section{RESULTADOS}

La tabla II muestra los resultados de los tres análisis de varianza comparando los tres grupos de aculturación en tres medidas de expectativas del paciente respecto del médico: 1) la puntuación total en la Escala de Expectativas del Paciente respecto del médico (EP); 2) las puntuaciones en la subescala de cuidado afectivo (CA), y 3) las puntuaciones en la subescala de tratamiento concreto (TC). Encontramos un efecto principal respecto de la 


\section{4}

TABLA II

Puntuaciones en el total de expectativas respecto del médico (EP), en Cuidado Afectivo (CA) y Tratamiento Concreto (TC) para los tres grupos de aculturación

\begin{tabular}{|c|c|c|c|c|c|c|}
\hline \multirow[b]{2}{*}{ Escala } & \multicolumn{3}{|c|}{ Medias no ajustadas } & \multicolumn{3}{|c|}{ Medias ajustadas ${ }^{2}$} \\
\hline & B & $\mathbf{M}$ & $\mathbf{A}$ & B & $\mathbf{M}$ & $\mathbf{A}$ \\
\hline $\begin{array}{l}\text { Expectativas respec- } \\
\text { to del médico }\end{array}$ & 34.50 & 32.00 & $30.58 *$ & 31.97 & 31.96 & 31.50 \\
\hline $\begin{array}{l}\text { Expectativas en cui- } \\
\text { dado afectivo }\end{array}$ & 9.17 & 8.61 & 8.97 & 8.31 & 8.47 & 9.16 \\
\hline $\begin{array}{l}\text { Expectativas en tra- } \\
\text { tam. concreto }\end{array}$ & 8.48 & 7.73 & 7.21 & 7.18 & 7.68 & 7.79 \\
\hline
\end{tabular}

Nota: Grupos de aculturación: $\mathrm{L}=$ bajo, $\mathrm{M}=$ medio, $\mathrm{H}=$ alto.

2 Puntuaciones ajustadas son las puntuaciones en las escalas una vez eliminados los efectos de la edad, estatus de salud y nivel de educación en EE. UU.

* $\mathrm{p}<.05$.

aculturación, basado en la EP, $\mathrm{F}(2,92)=3.47, \mathrm{p}<.05$. Las mujeres menos aculturadas tendían a esperar más del médico tal como lo indicaba su grado de puntuaciones medias en la EP, significativamente más altas. Las diferencias de grupo como una función del nivel de aculturación no fueron significativas para las subescalas CA y TC.

Sin embargo, a causa de las diferencias entre estos tres grupos de mujeres en edad, estatus de salud y en años de educación en EE. UU., las diferencias observadas en la escala EP aparentemente atribuibles al nivel de aculturación se confuden con los posibles efectos de estas tres variables. Las mujeres menos aculturadas pueden esperar más del médico porque son más viejas, están más enfermas o menos expuestas al sistema norteamericano de educación.

Para eliminar los efectos de estas variables, se realizó un análisis de covarianza para ver si los niveles más altos de expectativa respecto de los médicos se debían realmente a estas diferencias «culturales». El efecto significativo del estatus de aculturación (medido mediante la ARSMA) desapareció en un análisis de varianza cuando se eliminaron los efectos covariantes de la edad, estatus de salud y años de educación en EE. UU. Para el factor de aculturación, el análisis de covarianza arrojó $\mathrm{F}(2,71)=.045, \mathrm{p}<.05$.

Realizamos, igualmente, un análisis de regresión múltiple para ver si esperar más del médico (puntuación en la EP) estaba significativamente relacionado con alguna de estas covariantes: edad, estatus de salud, o nivel de educación en EE. UU. Los resultados de este análisis revelaron que el nivel de educación en EE. UU era la única variable que predecía significativamente la puntuación en la escala EP, $F(1,76)=4.07$, $p<.05$. Aparentemente, las mayores expectativas respecto del médico exhibidas como una función aparente de aculturación, estaban de hecho mediadas por la covariante de nivel de educación en EE. UU. Las mujeres con menor nivel de educación en EE. UU. esperaban más del médico.

Análisis de los ítems

Las puntuaciones individuales en los ítems de la escala EP fueron examinados de forma similar a través de los tres niveles de aculturación para 
obtener un analisis más detallado de las expectativas específicas respecto de los médicos en cuanto podían estar relacionadas con el nivel de acultura-

\section{TABLA III}

Ordenación de los items de la escala de expectativas en la consulta médica según la fuerza de las expectativas

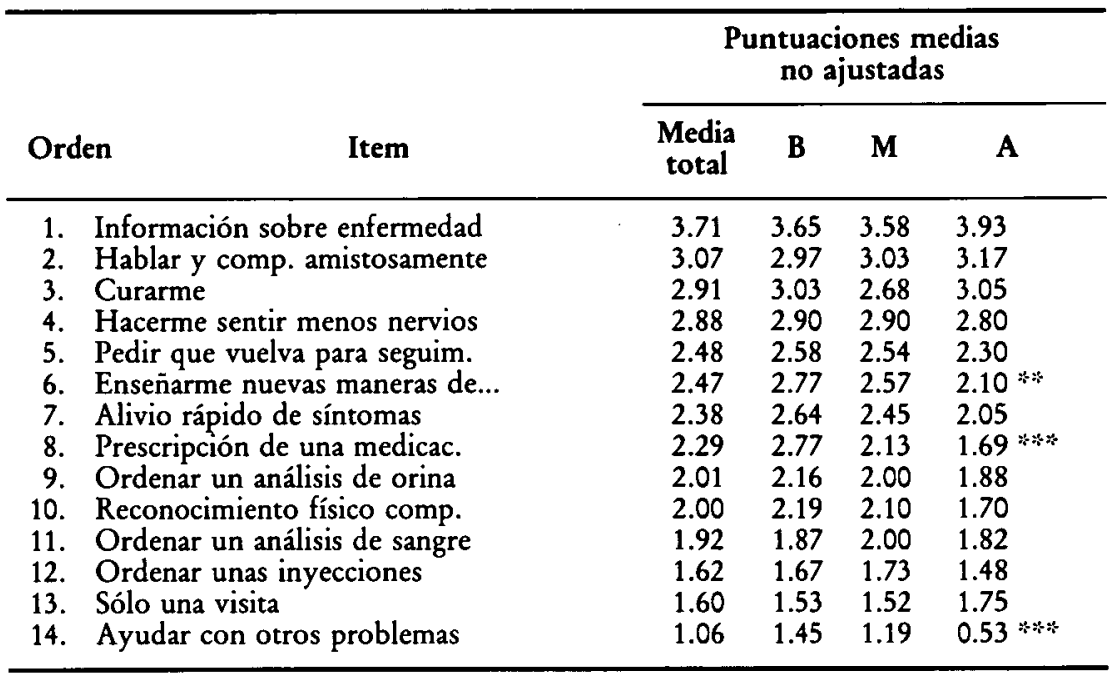

ción. La tabla III muestra los resultados de un análisis de varianza por cada ítem. Encontramos que tres items eran indicativos de diferencias de grupo significativas en las expectativas respecto del médico. Se observó inicialmente un efecto principal del factor de aculturación para las expectativas de que el médico: 1) «Me de una receta para un medicamento», $F(2,97)=$ $7.00, \mathrm{p}<.001 ; 2)$ «Me ayude con otros problemas además de mi enfermedad», $F(2,97)=6.87, p<.01$, y 3$)$ «Me enseñe nuevas maneras de tratar mis problemas», $\mathrm{F}(2,96)=4.56, \mathrm{p}<.01$. Los valores medios del grupo para cada uno de estos ítems sugieren que las mujeres de aculturación más baja tienen expectativas más fuertes de que el médico realice estas actividades.

Realizamos un análisis de covarianza para paliar los efectos confundentes de las tres convariantes ya mencionadas. Las puntuaciones medias ajustadas para los tres grupos de aculturación permanecieron significativamente diferentes sólo para el último de estos tres ítems: «Cuando voy a ver al médico porque me siento enfermo, espero que el médico me enseñe nuevas maneras de tratar mis problemas», $F(2,74)=3.40, \mathrm{p}<.05$. (Ver tabla III). Las puntuaciones medias ajustadas para este ítem indican que las mujeres mejicanas de baja aculturación esperan que se les enseñen nuevas maneras de tratar sus problemas «a menudo" $(M=2.92)$, mientras que las méjicoamericanas de aculturación media esperan esto entre «algunas veces» $\mathrm{y}$ «a menudo» $(M=2.59)$, y las mujeres anglo-americanas de alta aculturación esperan esto «algunas veces» y «a menudo» $(M=2.59)$, y las mujeres anglo-americanas de alta aculturación esperan esto «algunas veces" $(M=2.05)$. 
TABLA III (cont.)

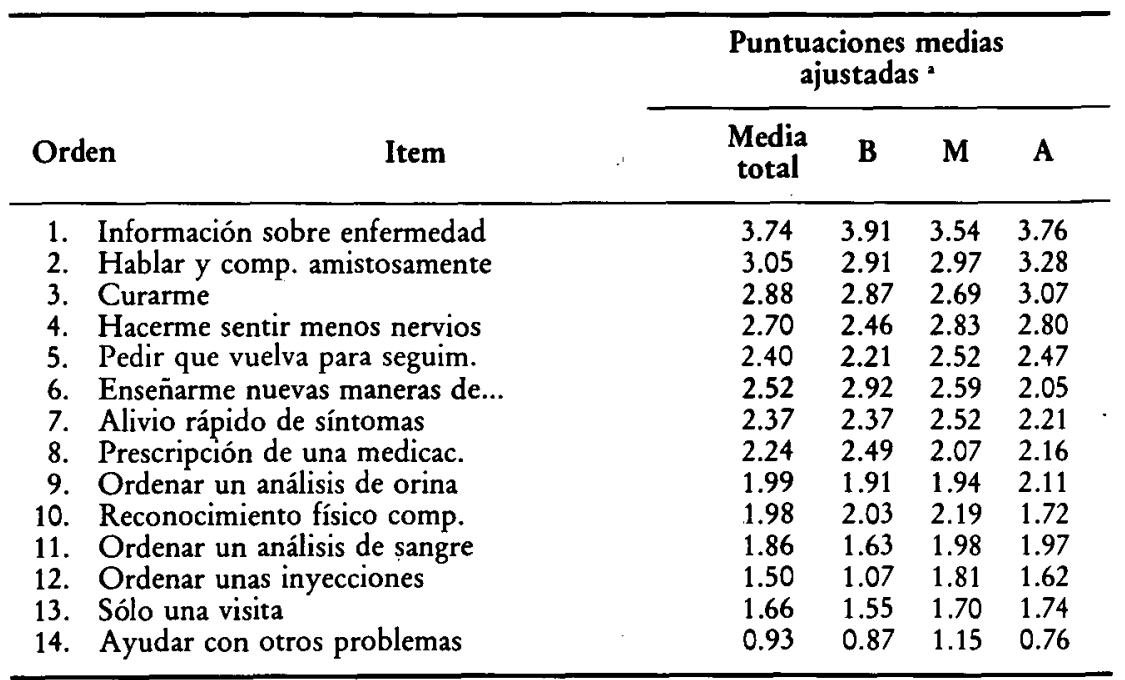

Nota: Las puntuaciones son: $(4)=$ casi siempre, (3) = a menudo, $(2)=$ algunas veces, $(1)=$ raras veces, (0) = nunca.

Las puntuaciones ajustadas son las puntuaciones obtenidas en las escalas una vez eliminados los efectos de la edad, el estatus de salud y el nivel de educación en EE. UU.

Finalmente, los ítems de la Escala de Expectativas (EP) fueron ordenados de acuerdo con las puntuaciones medias para los tres grupos de mujeres para identificar cuáles de estas mujeres esperaban más de su médico. La tabla III muestra esta ordenación según puntuaciones medias no ajustadas y según puntuaciones medias ajustadas al efecto de la edad, el estatus de salud y el nivel de educación en EE. UU. Sólo los ítems 5 y 6, y 12 y 13 fueron intercambiados en el orden cuando los ordenamos según las puntuaciones medias ajustadas.

Así, las mujeres que esperan más de su médico, indicaban que «siempre" desean que el médico les proporcione información sobre su enfermedad $(M=3.71)$ en una escala en la que la puntuación máxima es de 4 (= «siempre»). De forma semejante, estas mujeres «a menudo» desean que el médico tenga tiempo para ser amable y amistoso $(M=3.05)$ para curarlas $(M=2.88)$, para ayudarles a sentirse menos nerviosas con su enfermedad $(M=2.52)$ y. para concertar una nueva cita, si es necesaria $(M=2.40)$. Por el contrario, estas mujeres "rara vez" desean ser vistas una sola vez en el caso de estar enfermas $(M=1.66)$, que les pongan inyecciones $(M=1.50)$, o que se les ayude con otros problemas, además del de su enfermedad $(\mathrm{M}=0.93)$.

\section{DISCUSION}

El resultado más interesante en este trabajo fue la ausencia de diferencias interculturales en lo que estas mujeres esperaban de su médico, contra lo que sugería un modelo tradicional cultural-mejicano dibujado en la bibliografía sobre salud en méjico-americanos (Schreiber y Homiak, 1981). De forma inversa, también hay que notar el consenso relativo entre todas 
estas mujeres en su ordenación de expectativas respecto del médico, a despecho de las diferencias significativas entre ellas en nivel de aculturación, edad, estatus de salud, nivel de educación, lenguaje hablado y otras diferencias. Aunque dentro del grupo de aculturación (tabla III) se dieron algunas diferencias en la ordenación de los ítems de la EP, tales diferencias eran pequeñas.

La bibliografía sobre salud que describe las expectativas culturales de los méjico-americanos ha señalado que estas personas de minorías étnicas, particularmente las que tienen un bajo nivel de aculturación, a menudo esperarian del médico: 1) que pase mucho tiempo hablando y mostrándose amable; 2) que les de una receta para un medicmento; 3 ) que les prescriba inyecciones, 4) que les proporcione una cura $o$, al menos, 5) que les proporcione un alivio rápido de sus síntomas (Schreiber y Homiak, 1981; Hartwood, 1981). El grupo de mujres de baja aculturación de este estudio, que son las que más probablemente deberían exhibir tales expectativas, esperaban del médico más que las mujeres más aculturadas. Sin embargo, esta expectativa mayor desaparecía cuando se controlaban estadísticamente la edad, el estatus de salud y el nivel de educación. Este resultado particular sugiere que muchas pautas de conducta observadas clínicamente, que podían ser atribuibles a la "cultura" o a la "etnicidad", pueden ser mediadas por otros factores, tales como la edad, el estatus de salud o en nivel educativo.

De forma semejante, la sugerencia que aparece en la bibliografía al respecto de que es más probable que las mujeres de baja aculturación esperen cuidados afectivos o tratamientos puntuales que lo hagan las mujeres de - aculturación más alta, no se vio apoyada por los resultados de este trabajo, cuando estas expectativas fueron medidas mediante las subescalas de cuidado efectivo (CA) y de tratamiento concreto (TC). Estas mujeres de baja aculturación no insistieron más que las más aculturadas en sus expectativas respecto de que el médico pasara tiempo hablando y mostrándose amable, le ayudara a reducir su ansiedad, ni le proporcionara una cura. Tampoco esperaron con más frecuencia que se les proporcionaran medicamentos o inyecciones, ni que se les tomaran muestras de sangre $u$ orina.

Aunque las diferencias interculturales esperadas fueron pocas, esto no contradice el hecho de que hay ciertas acciones que estas mujeres, como conjunto, suelen esperar del médico. En la tabla III, los ítems 2, 3 y 4 son ítems de la subescala de Cuidado Afectivo (CA). Las altas puntuaciones de estos ítems sugieren que todas estas mujeres «a menudo" esperan que el médico les proporcione cuidado efectivo, como parte del cuidado usual de salud. Esta expectativa ha de ser contrastada con las puntuaciones comparativamente bajas de los ítems de la subescala de Tratamiento Concreto (TC) que estaban situadas en los puestos 8, 9, 11 y 12 en la tabla III. Aunque tales tratamientos concretos pueden esperarse a veces, la información y el cuidado afectivo son considerados por estas mujeres como formas mucho más importantes del cuidado médico.

Las expectativas con puntuación más alta (ver tabla III) implican, por parte de los pacientes, peticiones de información, de cuidado afectivo, de continuación de las citas para un seguimiento de su situación, de que se les enseñen nuevas maneras de tratar su enfermedad. Estas expectativas son congruentes con una perspectiva de promoción de la salud en el cuidado 


\section{8}

médico opuesta al «modelo médico tradicional», en el cual el paciente acepta pasivamente el tratamiento del médico. El hecho de que incluso las mujeres de baja aculturación tuvieran una ordenación similar en aquellas expectativas sugiere que todas estas mujeres están preparadas de forma semejante para participar en una relación dinámica médico-paciente en la que se proporciona información sobre salud-enfermedad y en la que la paciente puede participar en un programa de auto-cuidado y de cambio de conductas de salud, acciones que son consistentes con una perspectiva de promoción de la salud (Faber, 1982), y que deberían alentar al médico para proporcionar tal tipo de cuidados cuando y dondequiera sea posible. Los servicios de cuidado médico que podrían satisfacer las demandas hechas por estas mujeres consistirían en: información y apoyo efectivo, seguimiento del tratamiento e instrucción en nuevas formas de tratar con la enfermedad y mejorar su salud, intervenciones todas ellas consistentes con una perspectiva de promoción de salud. El médico de medicina general, o el médico familiar, es quizá el que está en mejor situación para alentar, organizar y guiar una intervención de mantenimiento de la salud y cambios de conductas no saludables. Sin embargo, si el médico está limitado en esa capacidad por falta de tiempo o falta de experiencia en los métodos de cambio de conducta, la experiencia de los psicólogos de la salud, o de los educadores de salud pública, puede ser utilizada en conjunción con la del médico para proporcionar a tales pacientes los cuidados médicos que maximicen verdaderamente la prevención de la enfermedad y la promoción de la salud.

La ausencia de diferencias culturales significativas observadas en este estudio no indica que la etnicidad y la aculturación no sean factores importantes en la consideración de las necesidades del cuidado de la salud de persona procedentes de minorías étnicas. Los efectos de ambos factores pueden ser potentes en relación con ciertas variables, aunque lo sean menos con relación a otras. Así, por ejemplo, Castro, Furth y Karlow (1984), en un estudio de características similares a este, encontraron diferencias en cuanto a la aceptación de la teoría de la oposición frío-caliente, y en las creencias biomédicas referidas a enfermedades cardiovasculares y a la relación entre estrés y enfermedad, relacionadas significativamente con el nivel de aculturación. Asimismo, encontraron que las mujeres menos aculturadas tenían un nivel significativamente más bajo de responsabilidad y control sobre su propia salud, caracterizado por una mayor aceptación de agentes externos de poder (Dios, la suerte, el médico, etc.). La investigación futura debe identificar claramente cuándo son importantes las diferencias de aculturación y cuándo no lo son. Relacionada con ello está la importancia de clarificar cuándo una diferencia observada se debe a la etnicidad, y cuándo se debe a variables correlacionadas con la etnicidad pero conceptualmente diferentes a ella, tales como la edad, el estatus socioeconómico, el nivel educativo, etc.

Este trabajo está limitado por el tamaño de la muestra utilizada, comparativamente pequeña, y por la inclusión de mujeres de procedencia urbana de diferentes niveles de aculturación. La falta de diferencias en relación con la variable de aculturación que se da en este estudio puede que no ocurra en otro estudio similar en el que se comparen grupos de mujeres de procedencia rural, con diferentes niveles de aculturación, con grupos em- 
parejados de mujeres de procedencia urbana. A pesar de tener un bajo nivel de aculturación, las mujeres urbanas pueden tener una exposición común a medios de comunicación de masas y otras fuentes de información en un marco urbano. $Y$ sobre todo, pueden compartir un mismo modelo de creencias respecto a la salud, a la enfermedad y a las expectativas de rol en la interacción médico-paciente (quizá, también en gran parte, determinado por la subcultura urbana). Ello puede asemejarlas de alguna manera respecto de sus conocimientos médicos y de sus expectativas respecto de la actuación del médico en la consulta, diferenciándolas claramente de las personas que viven en comunidades de algún modo aisladas del contacto con los medios de comunicación de masas y con fuentes diferentes de información médica y de influencia social.

\section{Referencias}

AcostA, F. X. (1980). «Self-described reasons for premature termination of psychotherapy by Mexican American, Black American and Anglo American patients». Psych. Reports, 47:435-443.

AJZEN, I. y FISHBEIN, M. (1980). Understanding attitudes and predicting social behavior. New Jersey: Prentice Hall.

ARCE, A. A. y TORRES-MATRULlO, C. (1982). «Application of cognitive behavioral techniques in the treatment of Hispanic patients». Psych. Quarterly, 54:230-236.

BOCHNER, S. (1983). «Doctor, Patients and their cultures». In D. Pendleton y J. Hasler (eds.). GDoctor-Patient Communication, New York: Academic Press, pp. 127-138.

BRISLIN, R. W. (1970), «Back translation for cross-cultural research».J. Cross-cultural Psych., $1: 185-216$.

CASTRO, F. G.; FurTh, P. y Karlow, H. (1984). «The Health Beliefs of Mexican, Mexican American and Anglo American Women». Hispanic J. of Behavioral Sciences, 6 (4):365-383.

Cuellar, I.; HaRris, L. C. y JASSO, R. (1980). «An acculturation scale for Mexican American normal and clinical population*. Hisp. J. Beb. Sci., 2:199-217.

Cuellar, I.; Martinez, C.; Jimenez, R. y GonZalez, R. (1983). «Clinical psychiatric case presentation: Culturally responsive diagnostic formulation and treatment in an Hispanic femalew. Hisp. J. Beb. Sci., 5:93-103.

CURRIE, B. F. y BeAsLeY, J. W. (1982). «Health Promotion in the medical encounter». In Health Promotion: Principles and clinical applications. (Edited by R. B. Taylor et al.), p. 143. Appleton-Century-Crofts, Norfork, Connecticut.

DAVIS, M. S. (1966). "Variations in patients' compliance with doctor' orders». J. of Medical Education, 19:263-279.

Del VeCCHIO GOOD, M. J.; GOOD, B. J. y NAsSI, A. J. (1983). «Patient requests in primary health settings: Development and validation of a research instrument». J. of Beh. Med., 6:151-168.

DimatTeO, M. R. y Dinicola, D. D. (1982). Achieving compliance: The Psychology of the Medical Practitioner's role. New York: Pergamon Press.

FABER, M. M. (1982). «Sharing responsability: The new health care partnership». In Promoting bealth through risk reduction. (Edited by M. M. Faber and A. M. Reinhart), p. 336. New York: Macmillan Publishing Co.

HARWOOD, A. (1981). Ethnicity and medical care. Cambridge, Mass.: Harvard University Press.

JAsParS, J.; KInG, J. y Pender?on, D. (1983). «The Consultation: A social psychological analysisw. In D. Pendleton y J. Hasler (eds.). Doctor-Patient Communication, London: Ácademic Press, pp. 139-157.

KOOPMAN, C.; EISENTHAL, S. y STOECKEL, J. D. (1984): «Ethnicity in the reported pain, emotional distress and requests of medical outpatients». Soc. Sci. Med., 18:487-490.

LEIGH, J. P. (1983). "Direct and indirect effects of education on health". Soc. Sci. Med., 17:227-234.

LURIE, H. J. y LAWRENCE, G. L. (1971). «Communication problems between rural Mexican American patients and their physicians». Am. J. of Ortbopsy., 42:777-783.

MARIN, G. Y TRIANDIS, H. C. (1984). Allocentrism as an important characteristic of the bebavior of Latin Americans and Hispanics. Paper presented at XXIII Congress of Psychology, Acapulco, Mex., Sept. 
Mechanic, D. (1968). Medical Sociology. New York: The Free Press.

Mollerman, E. KRabBendaM, P. J., AnNYAS, A. A. et al. (1984). "The significance of the doctor-patient relationship in coping with cancer». Soc. Sci. Med., 18:475-480.

NYAZEMA, N. Z. (1984). .Towards better patient drug compliance and comprehension: A challenge to medical and pharmaceutical services in Zimbabwe». Soc. Sci. and Med., 18:551-554.

PADILLA, A. M. (ed.) (1980). Acculturation: Theory, models and some new findings, Boulder, Colorado: Westview Press.

PAdilla, A. M. y LindHOLM, K. J. (1984). «Hispanic behavioral science research: Recommendations for future research». Hisp. J. Beb. Sci., 6:13-32.

Parsons, T. (1951). The Social System. Glencoe, Ill: The Free Press, pp. 428-479.

PARSONS, T. (1958). «Definitions of health and illness in the light of American values and social structurem. In E. G. Jaco (ed.): Physicians, Patients and Illness. New York: The Free Press, pp. 165-187.

PARSONS, T. (1975). "The Sick Role and the role of the physician reconsidered". Millbank Memorial Fund. Quarterly, 53:257-278.

RODIN, J. y JANIS, I. L. (1979). "The social power of health-care practitioners as agents of changew. J. of Social Issues, $35(1): 60-81$.

SCHREIBER, J. M. y HOMIAK, J. P. (1981). «Mexican Americans". In Ethnicity and medical care. (Edited by Harwood, A.), p. 264. Cambridge, Mass.: Harvard University Press.

STANDARD, K. y KAPLUN, A. (1983). «Health Education, new taks, new approaches». WHO Chronicle, 37:61-64.

SZASZ, T. S. y HOLLENDER, M. H. (1956). «A contribution to the philosophy of medicine: The basic models of the doctor-patient relationshipn. Arch. of Internal Medicine, 97:585-592.

TRIANDIS, H. C., KaShima, Y., HUi, H. et al. (1982): «Acculturation and biculturalism indices among relatively acculturated Hispanic young adults». Int. J. Psychol., 16:140-159.

TrotTER, R. T.. «Contrasting models of the healer's role: South Texas case examples». Hiso. J. Beh. Sci., 4:315-327.

TUCKETT, D. y ANTHONY, W. (1984). "Approaches to the measurement of explanation and information giving in medical consultations: A review of empirical studies". Soc. Sci. and Med., 18:571-580.

WEISS, S. J. y DAVIS, H. P. (1983). *The Health role expectations index: A measure of alignment, disparity and changen. J. of Beh. Med., 6:63-76.

WELLS, K.; WARE, J. E. y LEWIS, C. E. (1984). «Physicians' practices in counseling patients about health habits». Medical Care, 22:240-246.

Yamamoto, J.; Acosta, F. X., Evans, L. A. y STILbeck, W. M. (1984). «Orienting therapists about patients' needs to increase patient satisfaction.. Amer. J. Psychiatry, 141:274-277. 